\title{
Comprehensive investigation of defects in highly perfect silicon single crystals
}

\author{
I.V. Prokopenko' ${ }^{1}$ E.N. Kislovskii ${ }^{2}$, S.I. Olikhovskii², V.M. Tkach ${ }^{3}$, P.M. Lytvyn¹, \\ T.P. Vladimirova ${ }^{2}$ \\ ${ }^{1}$ Institute of Semiconductor Physics, NAS Ukraine, 45 Prospect Nauky, Kyiv-28, UA-03650, Ukraine \\ Tel.: (380-44) ; Fax: (380-44) ; E-mail: \\ ${ }^{2}$ G.V. Kurdyumov Institute of Metal Physics, NAS Ukraine, 36 Blvd. Vernadskogo, Kyiv-142, UA-03680, Ukraine \\ ${ }^{3}$ Institute of Super-Hard Materials, NAS Ukraine, Kyiv, Ukraine
}

\begin{abstract}
We used X-ray diffraction method of total rocking curves and nondestructive direct observation techniques (atomic force and scanning electron microscopies) to quantitatively determine the defect characteristics (radii and concentrations) for the main types of defects in Czochralski-grown silicon single crystals annealed at $750^{\circ} \mathrm{C}$.
\end{abstract}

Keywords: X-ray diffraction, structural defects, silicon, scanning electron microscopy, atomic force microscopy.

Paper received 09.06.00; revised manuscript received 04.07.00; accepted for publication 12.07.00.

\section{Introduction}

It is well known that silicon single crystals without dislocations, even the most perfect ones, contain different structural defects. These are point defects (interstitial silicon atoms, vacancies and impurity atoms) and various clusters formed by them [1,2]. Characterization of defect structure in such crystals is of great importance for modern silicon-based instruments making [1,2] and solving numerous problems in standardization and metrology $[3,4]$, as well as for fundamental investigations of crystalline structure $[5,6]$.

However, the above problem of characterization of defects and structural imperfections still remains far from being solved. This is due to the fact that defects induce extremely small lattice distortions, and it is very difficult to detect them. To illustrate, one practically cannot detect defects in Czochralski-grown silicon single crystals (annealed at $750^{\circ} \mathrm{C}$ ) by using X-ray topography (see, e.g., [2]). To do this, some special conditions are to be provided, such as high-resolution plane-wave $\mathrm{X}$-ray topography by using synchrotron radiation for very thin samples [7] (see also [8]). The conventional X-ray diffractometry, when being applied to such samples, enables one to detect imperfections only at measurements of integrated intensities $[9$, 10]. Their analysis makes it possible to determine only some general parameters of structural perfection - static Debye-Waller factor and defect correlation length, and, at best, to determine parameters for defects of only one type that is assumed to be predominant.

True, for silicon samples annealed at $750{ }^{\circ} \mathrm{C}$ the integral and differential $\gamma$-diffractometries $[11,12]$ enable one to detect very large deviations from intensities characteristic for perfect crystals. These techniques (and smallangle neutron scattering $[12,13]$ as well), however, have not found a wide practical utility. Besides, it is impossible to analyze successfully the results obtained by these methods without using an adequate theoretical model for dynamical diffraction of radiations in single crystals containing defects. And the complicated character of actual defect structure also has to be accounted for.

The above difficulties may be overcome to a large extent by applying the total rocking curve (TRC) technique, with the use of high-resolution double-crystal X-ray diffractometry $[14,15]$. This method is based on the generalized dynamical theory of radiation scattering by imperfect crystals. It gives a self-consistent description for angular dependencies of both coherent and diffuse TRC components [16]. The method employs explicit analytical relationships between the determined diffraction parameters of structural perfection (the static Debye-Waller factor and coefficient of absorption due to diffuse scattering) and defect characteristics. This makes it possible to perform comprehensive quantitative diagnostics of actual defect structures in single crystals. 


\section{I.V. Prokopenko et al.: Comprehensive investigation of defects in highly perfect ...}

The objective of this work was to make comprehensive investigation of actual complex defect structure for highly perfect Czochralski-grown silicon single crystals, both non-annealed and after isothermal annealing at $750^{\circ} \mathrm{C}$. To do this, we used the above TRC technique and highresolution nondestructive direct observation methods (atomic force and scanning electron microscopies).

\section{Investigation techniques}

\subsection{Total rocking curve technique for Bragg diffraction}

The TRC technique is a generalization (for the case of large defects) of the well-known method of «integral» diffuse scattering intensity (Larson method - see [17, 18]). Later based on kinematical description of the integrated (over the output angles) diffuse scattering intensity at the TRC tails. Such an approach makes it possible to determine both defect size and concentration $n$ from the dependence of the integrated diffuse scattering intensity on the angle of incidence. If the effective sizes of defects $R_{\text {eff }}$ are comparable to (or exceed) the extinction length $\Lambda$, then one has to consider jointly the coherent and diffuse components of scattering intensity in the whole (including total reflection range) angular on the basis of dynamical theory.

When the measurements are made by using the double-crystal diffractometer with widely open detector window, TRC for a single crystal where defects are uniformly distributed is a sum of two (coherent and diffuse) diffraction intensity components that are integrated over the output solid angle. They depend on sample deviation $\Delta \theta$ from the exact Bragg reflection position of the crystal studied $[14,15]: R(\Delta \theta)=R_{\text {coh }}(\Delta \theta)+R_{\text {diff }}(\Delta \theta)$.

Information on the defect characteristics appears in these components through the static Debye-Waller factor, $E=\exp \left(-L_{\mathbf{H}}\right)$, and coefficient of absorption due to diffuse scattering, $\mu_{\mathrm{ds}}(\Delta \theta)$. In this case, in the semiinfinite crystal approximation (i.e., when $\mu_{0} t>1$, where $\mu_{0}$ is the linear coefficient of photoelectric absorption and $t$ is the crystal thickness), the coherent component, $R_{\text {coh }}$, is given by the expression whose form is similar to that of the corresponding one for a perfect crystal [19], but with some additional parameters that are expressed through $E$ and $\mu_{\mathrm{ds}}$. For symmetric diffraction the diffusion component is (in the same approximation)

$$
R_{\text {diff }}(\Delta \theta)=\frac{\gamma_{0} \mu_{\mathrm{ds}}(\Delta \theta)}{2 \mu(\Delta \theta)} .
$$

Here $\gamma_{0}$ is the direction cosine of the incident X-ray beam; the interference absorption coefficient, $\mu(\Delta \theta)$, is of the order of $\pi / \Lambda$ in the total reflection range, and is about $\mu_{0} / \gamma_{0}$ at $|\Delta \theta| \gg>w$, where $w$ is the width of the total reflection range.

The angular dependence of $\mu_{\mathrm{ds}}$ is very sensitive to defect type and size. This fact enables one to perform diag- nostics of complex defect structures formed by defects of different types and sizes [20,21]. If the defect concentrations and sizes are sufficiently small, then the superposition principle for their static displacements fields holds [22]. In this case, when there are no correlations in the defect positions, one can present the general diffraction parameters of structural perfection, $L_{\mathbf{H}}$ and $\mu_{\mathrm{ds}}$, as sums of contributions from the defects of each type $\alpha$ [21]:

$$
L_{\mathbf{H}}=\sum_{\alpha} L_{\mathbf{H}}^{\alpha}, \quad \mu_{\mathrm{ds}}(\Delta \theta)=\sum_{\alpha} \mu_{\mathrm{ds}}^{\alpha}(\Delta \theta) .
$$

To perform adequate quantitative diagnostics of defects from the TRC angular dependencies $R(\Delta \theta)$ in such cases (and especially when the defect effective radius $R_{\text {eff }}$, for at least one type of defects, is comparable to the extinction length $\Lambda$ ), one has to measure TRC with high versus incidence angle resolution.

The incident X-ray beam has to be characterized by high degree of collimation and monochromatization. An additional reason for these requirements is that in this case one can detect intensity oscillations for asymptotic diffuse scattering [23-25, 21]. This substantially improves the diagnostics reliability. The period of these oscillations for diffuse scattering intensity (both differential [23-25] and integrated over output angle [21]) is determined by the effective defect radius $R_{\text {eff }}$. For clusters this radius is $R_{\text {eff }}=\sqrt{H\left|A_{c l}\right|}$. Here $H$ is the magnitude of reciprocal space vector; $A_{c l}$ is the cluster power (for spherical clusters $A_{c l}=\Gamma \varepsilon R_{P}^{3}$, where $\Gamma=\frac{1}{3}(1+v) /(1-v), v$ is the Poisson ratio, $\varepsilon$ is the strain at cluster boundary and $R_{P}$ is the cluster radius). For dislocation loops $R_{\text {eff }}=R_{L} \sqrt{H b}$, where $R_{L}$ is the loop radius and $b$ is the Burgers vector magnitude.

The above features of TRC technique, as well as conditions for diagnostics of complex defect structures, determine the use of double-crystal diffractometers with high-resolution X-ray optical design (see Fig. 1). To treat adequately the measured TRC, one has to take into account the instrumental factors. This can be done by single con-

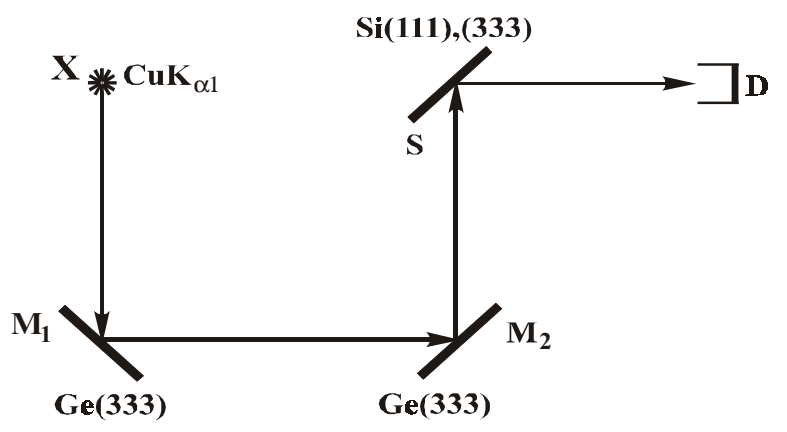

Fig. 1. Diagram of $\mathrm{Si}$ (111) and $\mathrm{Si}$ (333) symmetric Bragg reflections measurements by a high-resolution X-ray diffractometer $\left(\mathrm{Cu} K_{\alpha 1}\right.$ characteristic radiation): $\mathrm{X}-\mathrm{X}$-ray tube; $\mathrm{M}_{1}$ and $\mathrm{M}_{2}$ - monochromators; $\mathrm{S}$-sample; D-detector. 


\section{I.V. Prokopenko et al.: Comprehensive investigation of defects in highly perfect ...}

volution of the instrumental function with TRC of the crystal studied [20].

\subsection{Direct observation techniques: atomic force and scanning electron microscopies}

To study the sample surfaces, we used the atomic force microscope (AFM) Nanoscope IIIa (Digital Instruments, USA) and scanning electron microscope TESLA BS-340 (Czech Republic). The analytical system for energy-dispersive X-ray microanalysis LINK-860 (UK) enabled us to qualitatively determine distribution of phases and diffusion areas in the samples studied by using characteristic $\mathrm{X}$-ray radiation.

The AFM scans were taken by the $100 \mu \mathrm{m}$ scanner (Gscanner) using etched silicon tip (whose radius of curvature was $\sim 7 \mathrm{~nm}$, resonance frequency was $320 \mathrm{kHz}$ and spring constant was about $\sim 60 \mathrm{~N} / \mathrm{m}$ ) in tapping mode. This enabled us to study the sample surface features whose sizes varied from several Ångströms up to tens of microns.

\section{Experimental samples}

The samples used for investigations were prepared from wafers that have been cut from the central part of a Czochralski-grown silicon ingot (growth axis $\langle 111\rangle$ ). The material was of $p$-type; its resistivity was $10.5 \Omega \cdot \mathrm{cm}$. Concentrations of oxygen and carbon contaminations were $\sim 1.1 \cdot 10^{18}$ and $\leq 10^{17} \mathrm{~cm}^{-3}$, respectively. The samples, cut from wafers transversely to the growth axis, were exposed to chemico-dynamic treatment. After it their thickness was $500 \mu \mathrm{m}$. To remove possible markings of the treatment, the samples were further etched to the depth of $10 \mu \mathrm{m}$ on both sides.

The samples obtained in the above way were separated into three groups: L00, L30 and L50. Those from L30 and L50 groups were annealed at a temperature of $750{ }^{\circ} \mathrm{C}$ for 30 and 50 hours, respectively. (Thermal annealing was performed in a sealed-off quartz tube in the argon atmosphere, at a pressure of $150 \mathrm{kPa}$, to exclude possible oxygen «depletion» of the near-surface layer.) Then they were air-hardened and washed with hydrofluoric acid for two minutes to remove oxide film that might appear at the sample surface.

The L00 samples were not annealed. They were used for studies of defect structure in the as-grown single crystal.

\section{Results of direct observations}

Our investigations with the atomic force microscope have reveal substantial distinctions in surface morphology of the samples studied (see Fig. 2).

For as-grown L00 samples (Fig. 2a) there are pyramidal ridges randomly distributed over the surface. Their average size and height are about $0.1 \mu \mathrm{m}$ and $25 \mathrm{~nm}$, respectively. Their surface concentration is about $8 \cdot 10^{8} \mathrm{~cm}^{-2}$.

For annealed L30 samples (Fig. 2b) one can see pyramidal precipitates (whose average sizes are 0.2 and
$0.4 \mu \mathrm{m})$, as well as their aggregates, at the surface. The ridge height is about $50 \mathrm{~nm}$.

For annealed L50 samples (Fig. 2c) the average sizes of pyramidal ridges are about 0.12 and $0.45 \mu \mathrm{m}$, while their average heights are 10 and $50 \mathrm{~nm}$, respectively.

We also made an analysis of the sample surface roughness, $R_{a}$, based on the results given by the atomic force microscopy. To exclude the effect of possible surface macroscopic tilt on the roughness value, we calculated the surface roughness by the following expression:

$R_{a}=\frac{1}{L_{x} L_{y}} \int_{0}^{L_{y}} \int_{0}^{L_{x}}|f(x, y)| d x d y$.

Here $f(x, y)$ is a function that represents the deviation of the surface studied from a plane; $L_{x}$ and $L_{y}$ are sizes of the area for which one determines roughness.

It is remarkably that for annealed samples total surface roughness, $R_{a}^{\text {av }}$, is substantially below that for as-grown samples (see Table 1). At the same time the surface roughness $R_{a}$ (determined for the field $0.5 \times 0.5 \mu \mathrm{m}$ without macroscopic ridges) is approximately the same for all samples. It should be noted, however, that after thermal annealing for only 30 hours the surface roughness $R_{a}$ and nonuniformity are maximal. Thermal annealing for 50 hours leads to a roughness decrease and makes surface more uniform.

One can see from the scanning electron microscopy patterns taken for the L30 sample (Fig. 3a) that there are precipitates (about $0.5 \mu \mathrm{m}$ in size) and their aggregates. The characteristic X-ray radiation mapping over this surface (Fig. 3b) suggests that the above precipitates are inclusions of chromium atoms bonded to oxygen (probably $\mathrm{CrO}_{3}$ ). The scanning electron microscopy patterns taken for the L50 sample (Fig. 3c) show precipitates whose size is $\leq 1 \mu \mathrm{m}$, as well as linear defects (about $10 \mu \mathrm{m}$ in size) stemming from dislocation loops intersection with the crystal surface.

\section{X-ray diffraction studies and analysis of their results}

The TRC for the samples studied are shown in Fig. 4. When treating them, we used a model for defect structure that included presence of randomly distributed disk-like pre-

Table 1. Surface roughness for as-grown sample LOO and annealed samples L30, L50 $\left(\varepsilon, \%-R_{a}\right.$ deviation from its average value at different surface points).

\begin{tabular}{c|c|c|c}
\hline \multirow{2}{*}{$\begin{array}{c}\text { Sample } \\
\text { No }\end{array}$} & \multicolumn{2}{|c|}{$\begin{array}{c}\text { Field sizes } \\
0.5 \times 0.5 \mu \mathrm{m}\end{array}$} & $\begin{array}{c}\text { Field sizes } \\
5 \times 5 \mu \mathrm{m}\end{array}$ \\
\cline { 2 - 4 } & $R_{a}, \AA$ & $\varepsilon, \%$ & $R_{a}^{\mathrm{av}}, \AA$ \\
\hline L00 & 6.6 & 7.3 & 29.8 \\
\hline L30 & 6.9 & 22.7 & 17.6 \\
\hline L50 & 5.2 & 13.0 & 10.3 \\
\hline \hline
\end{tabular}


I.V. Prokopenko et al.: Comprehensive investigation of defects in highly perfect ...
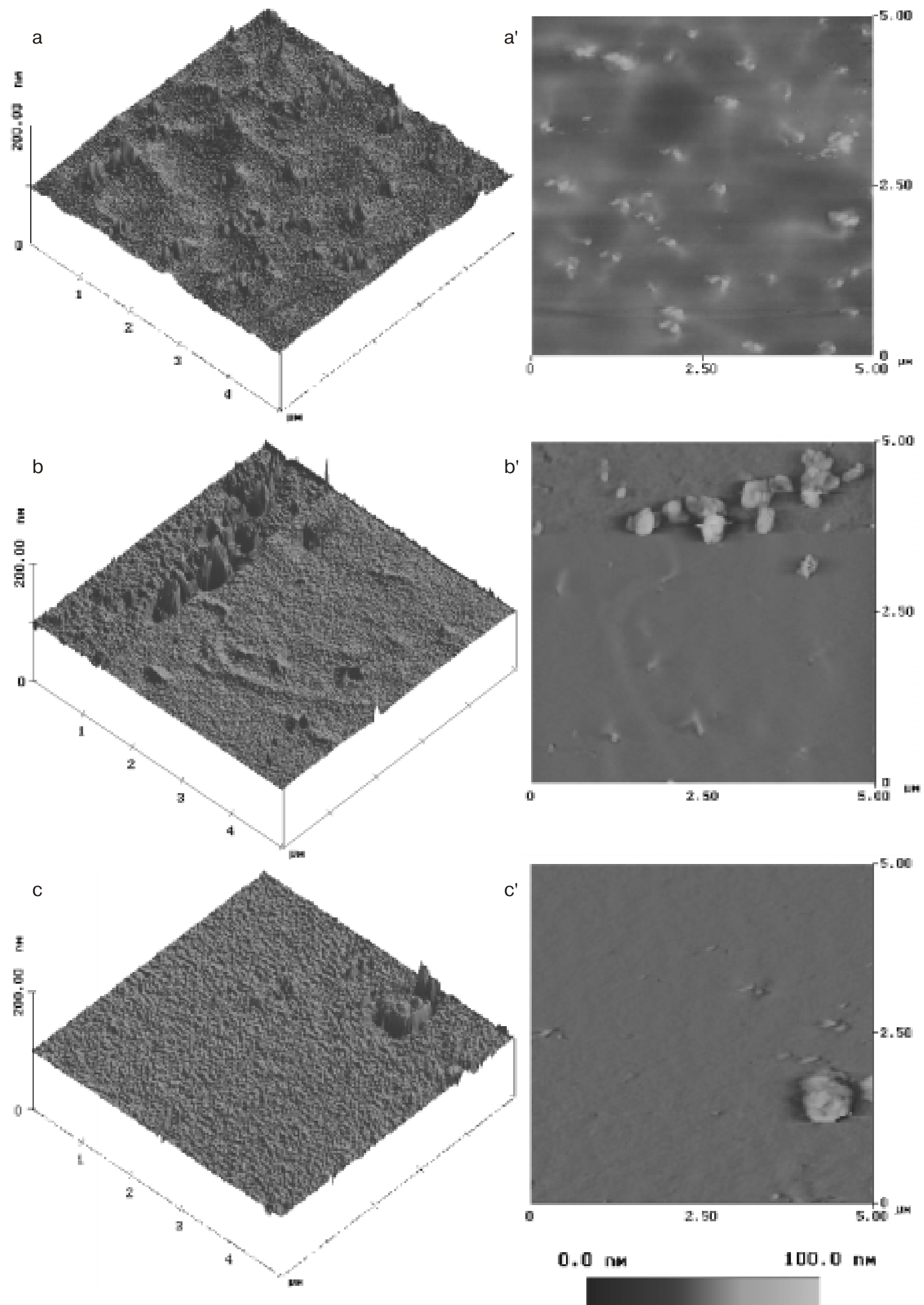

Fig. 2. Solid and plane patterns of Si sample surface relief given by the atomic force microscope for samples L00 (a, a'), L30 (b, b') and L50 (c, c'). 


\section{I.V. Prokopenko et al.: Comprehensive investigation of defects in highly perfect ...}

Table 2. Characteristics of precipitates and dislocation loops in the samples determined for Si (111) i (333) reflections (Cu $K_{\alpha 1}$-line). ( $R_{P}, h_{P}$ and $n_{P}$ are the radius, thickness and density of disk-like precipitates, respectively; $R_{L}$ and $n_{L}$ are the radius and density of dislocation loops, respectively.)

\begin{tabular}{|c|c|c|c|c|c|}
\hline $\begin{array}{l}\text { Sample No } \\
\text { and } h k l\end{array}$ & $R_{P}, \mu \mathrm{m}$ & $h_{P}, \mathrm{~nm}$ & $n_{P}, \mathrm{~cm}^{-3}$ & $R_{L}, \mu \mathrm{m}$ & $n_{L}, \mathrm{~cm}^{-3}$ \\
\hline \multicolumn{6}{|l|}{ L00 } \\
\hline 111 & 0.1 & 6.3 & $9.0 \cdot 10^{10}$ & 0.025 & $2.3 \cdot 10^{12}$ \\
\hline 333 & 0.1 & 6.3 & $7.0 \cdot 10^{10}$ & $\begin{array}{l}17.0 \\
0.025 \\
2.0\end{array}$ & $\begin{array}{l}3.5 \cdot 10^{11} \\
9.3 \cdot 10^{12} \\
1.4 \cdot 10^{8}\end{array}$ \\
\hline \multicolumn{6}{|l|}{ L30 } \\
\hline \multirow[t]{2}{*}{111} & 0.25 & 9.1 & $1.6 \cdot 10^{10}$ & 0.03 & $5.0 \cdot 10^{12}$ \\
\hline & 0.57 & 12.7 & $5.0 \cdot 10^{9}$ & 10.0 & $1.1 \cdot 10^{7}$ \\
\hline \multirow{2}{*}{333} & 0.25 & 8.3 & $1.7 \cdot 10^{11}$ & 0.03 & $7.5 \cdot 10^{12}$ \\
\hline & 0.57 & 12.7 & $5.0 \cdot 10^{9}$ & 2.0 & $1.2 \cdot 10^{8}$ \\
\hline \multicolumn{6}{|l|}{ L50 } \\
\hline 111 & 0.6 & 13.0 & $1.0 \cdot 10^{9}$ & 0.035 & $2.0 \cdot 10^{12}$ \\
\hline 333 & 0.6 & 13.0 & $7.8 \cdot 10^{9}$ & $\begin{array}{c}5.0 \\
0.035 \\
2.0\end{array}$ & $\begin{array}{l}5.0 \cdot 10^{7} \\
5.5 \cdot 10^{12} \\
1.5 \cdot 10^{8}\end{array}$ \\
\hline
\end{tabular}
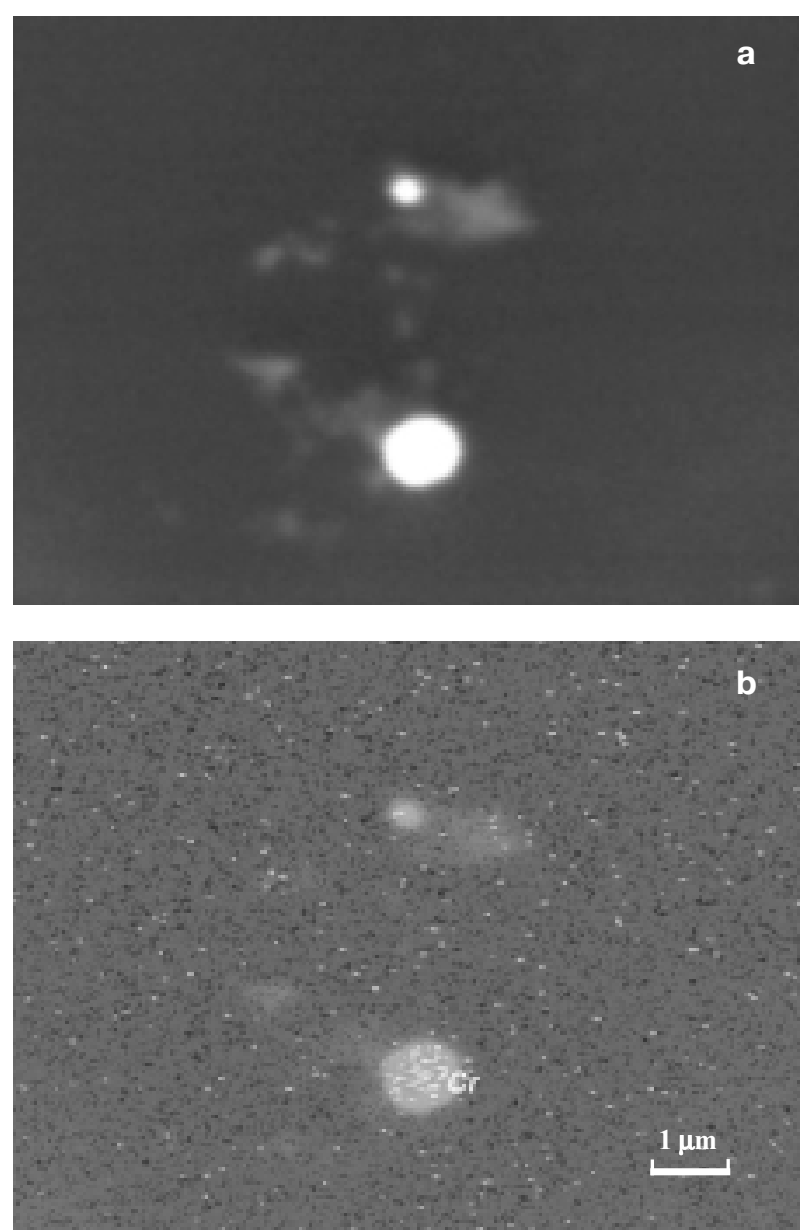

b

$S Q O, 3(3), 2000$ cipitates and circular dislocation loops in the crystal, as well as point defects and thermal diffuse scattering $[20,21]$. It should be noted that all attempts to describe the observed TRC in the whole angular range by using only one type of defects failed (it was possible to fit only isolated regions of angular dependencies). This results from the fact that diffuse components of TRC from defects of different types (and sizes) behave in different ways in different regions of the angular range. It is that fact

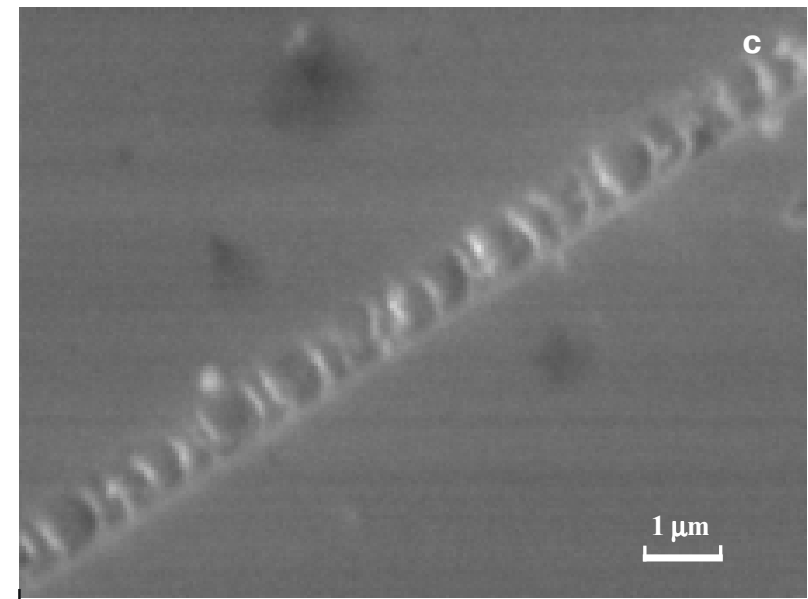

Fig. 3. Patterns of Si single crystal surface given by the scanning electron microscope for samples L30 (the image in scattered electrons (a), the same area but in characteristic X-ray radiation of chemical elements of surface (b)) and L50 (the image in scattered electrons (c)). 


\section{I.V. Prokopenko et al.: Comprehensive investigation of defects in highly perfect ...}
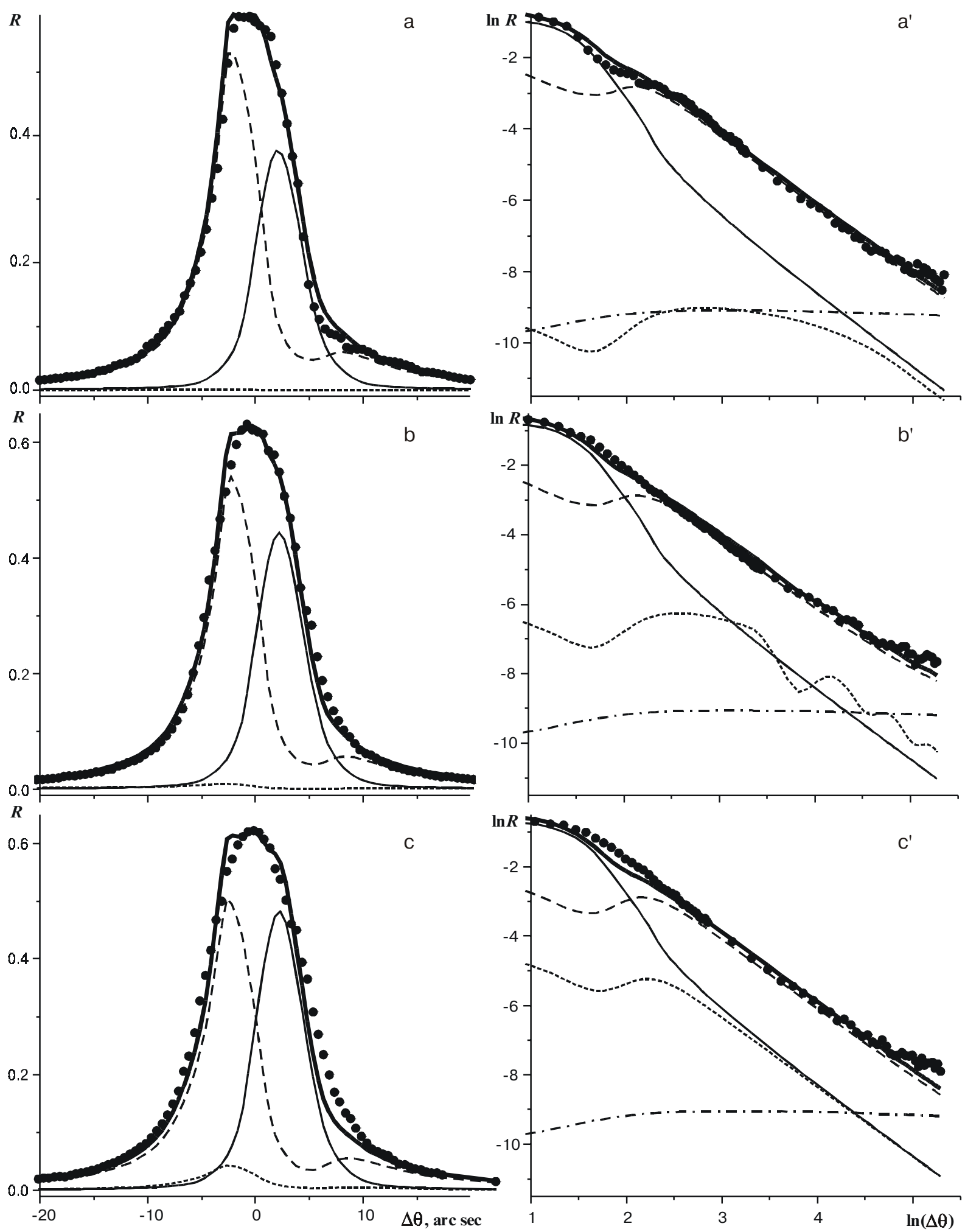

Fig. 4. Total rocking curves for Si samples ( $\mathrm{Cu} K_{\alpha 1}$ characteristic radiation, (111) reflection) in the total reflection range (a, b, c) and at tails ( $\left.\mathrm{a}^{\prime}, \mathrm{b}^{\prime}, \mathrm{c}^{\prime}\right)$ (solid line-theory, dots - experiment). Samples: L00 (a, a'), L30 (b, b') and L50 (c, c'). Shown are also the coherent (thin solid line) and diffuse TRC components for precipitates (dashed line), dislocation loops (dotted line) and point defects (dash-dotted line).

which enables one to perform diagnostics of complex defect structures. In this case a consistency of TRC description in the total reflection range (Bragg peak) and at the tails is of importance.
We determined the defect parameters (radius $R_{P}$, thickness $h_{P}$ and density $n_{P}$ of disk-like precipitates, radius $R_{L}$ and density $n_{L}$ of dislocation loops) by fitting the measured TRC with use of the expressions for $R_{\mathrm{coh}}$ and $R_{\text {diff }}$ 


\section{I.V. Prokopenko et al.: Comprehensive investigation of defects in highly perfect ...}

obtained in [20, 21]. The determined parameter values are given in Table 2 . One should note the agreement between the parameters values of precipitates and small loops found from TRC for different reflections. The sizes of large precipitates and dislocation loops agree well with those determined from direct observations (see Figs. 2 and 3). The sizes of small precipitates and dislocation loops are close to those observed for similar samples by transmission electron microscopy [26-28]. Differences between the densities may be due to different conditions of statistics investigation with X-ray diffractometry and electron microscopy, as well as different thermal prehistory of crystals and growing conditions. They essentially affect both the precipitation centers density and defect formation [2].

Attention is drawn to the complex defect structure of the as-grown L00 sample (see defect parameter values in Table 2). It should be noted that various defects in these crystals have been also observed by using plane-wave synchrotron X-ray topography [7]. Comparison between the defect parameter values obtained for the as-grown (L00) and annealed (L30 and L50) samples enables one to state that the initial defect structure substantially affects the character of defect production during further thermal treatments.

We would also like to stress that TRC technique makes it possible to perform quantitative diagnostics of diffraction parameters for point defects and thermal diffuse scattering $\left(\mu_{\mathrm{ds}}(0) / \mu_{0}\right.$ is equal $3.2 \cdot 10^{-4}$ for (111) reflections and $5.6 \cdot 10^{-5}$ for (333) reflections)(see Fig. 4). It should be noted that both the measurements and analysis of such low intensities of diffuse scattering in the immediate vicinity of the reciprocal lattice point are only possible due to separation of much higher contributions to diffuse scattering from microdefects.

\section{Conclusions}

We have made a comprehensive investigation of defect structure for Czochralski-grown silicon single crystals annealed at $T=750^{\circ} \mathrm{C}$. Direct observations by atomic force and scanning electron microscopes enabled us to determine the main types of defects in the above highlyperfect crystals. We also measured TRC for these crystals by high-resolution double-crystal diffractometer and performed an analysis of the results obtained. In doing this we based on the generalized dynamical theory of radiations scattering by imperfect single crystals. This enabled us to determine numerical values of the defect characteristics, as well as of diffraction parameters of diffuse scattering from point defects and thermal diffuse scattering.

The results obtained and their comparison to the reference data for similar samples that were obtained using destructive methods of direct observation evidence that it is possible to perform reliable and highly informative di- agnostics of complex defect structures in single crystals by combination the non-destructive methods of direct observation and X-ray differential technique of total rocking curve.

\section{References}

1. C. Claeys, J. Vanhellemont // J. Cryst. Growth 126, p. 41 (1993).

2. A. Borghesi, B. Pivac, A. Sassella, A. Stella // J. Appl. Phys. 77(9), p. 4169 (1995).

3. J. Härtwig, G. Hölzer, E. Föster, K. Goetz, K. Wokulska, J. Wolf // Phys. stat. sol. 143(1), p. 23 (1994).

4. R.D. Deslattes, E.G. Kessler, S. Owens, D. Black, A. Henins // J. Phys. D: Appl. Phys. 32(4A), p. A3 (1999).

5. M. Deutsch, M. Hart, S. Cummings // Appl. Phys. Lett. 51, p. 1410 (1987)

6. J. Vanhellemont // J. Cryst. Growth 180, p. 111 (1997).

7. Y. Chikaura, M. Imai, Y. Suzuki, Y. Yatsurugi // J. Cryst. Growth 103, p. 141 (1990).

8. T. Ishikawa // J. Phys. D: Appl. Phys. 28(4A), p. A256 (1995).

9. S.N. Voronkov, D.N. Piskunov, F.N. Chukhovskii, S.K. Maksimov // ZhETF 92(3), p. 1099 (1987).

10. S.N. Voronkov, F.N. Chukhovskii, P.V. Lomovtsev, S.P. Darbinyan // Phys. stat. sol. (a) 134(2), p. 301 (1992).

11. J.R. Schneider, R. Bouchard, H.A. Graf, H. Nagasawa // Acta Crystallogr. A 48(6), p. 804 (1992).

12. R. Bouchard, J.R. Schneider, S. Gupta, S. Messoloras, R.J. Stewart, H. Nagasawa, W. Zulehner // J. Appl. Phys. 77(2), p. 553 (1995).

13. F.M. Livingston, S. Messoloras, R.C. Newman, B.C. Pike, R.J. Stewart, M.J. Binns, W.P. Brown, J.G. Wilkes // J. Phys. C: Solid State Phys. 17(34), p. 6253 (1984).

14. V.B. Molodkin, S.I. Olikhovskii, E.N. Kislovskii, V.P. Krivitskii, A.V.Los', E.V. Pervak, J.E. Ice, B.K. Larson // Metallofizika i Noveishie Tekhnologii 19(12), p. 25(1997).

15. V.V. Nemoshkalenko, V.B. Molodkin, S.I. Olikhovskii, E.N Kislovskii, V.P. Krivitskii, E.G. Len', E.V. Pervak, J.E. Ice, B.K. Larson, Dynamic interpretation of total rocking curve for Bragg X-ray diffraction by single crystals with microdefects (in Russian), Preprint UNSC 2, Kiev (1998).

16. L.I. Datsenko, V.B. Molodkin, M.E. Osinovskiii, Dynamic X-ray Scattering by Actual Crystals (in Russian), Naukova Dumka, Kiev (1988).

17. P.H. Dederichs // Phys. Rev. B 4(4), p. 1041 (1971).

18. B.C. Larson // J. Appl. Cryst. 8(2), p. 150 (1975).

19. Z.G. Pinsker, X-ray Crystal Optics (in Russian), Nauka, Moscow (1982).

20. S.I. Olikhovskii, E.M. Kislovskii, V.B. Molodkin, E.G. Len', T.P. Vladimirova, O.V. Reshetnik // Metallofizika i Noveishie Tekhnologii 22(6), p. 3 (2000).

21. E.M. Kislovskii, S.I. Olikhovskii, V.B. Molodkin, E.G. Len', T.P. Vladimirova // Metallofizika i Noveishie Tekhnologii 22(7), p. 3 (2000).

22. M.A. Krivoglaz, $X$-ray and Neutron Diffraction in Non-Ideal Crystals (in Russian), Naukova Dumka, Kiev (1983).

23. H. Trinkaus // Z. Angew. Phys. 31(5), p. 229 (1971).

24. H. Trinkaus, H. Spalt, H. Peisl // Phys. stat. sol. (a) 2(1), p. K97 (1970).

25. P. Ehrhart, H. Trinkaus, B.C. Larson // Phys. Rev. B 25(1), p. 834 (1982).

26. K. Wada, N. Inoue, K. Kohra // J. Cryst. Growth 49, p. 749 (1980).

27. W. Bergholz, M.J. Binns, G.R. Booker, J.C. Hutchison, S.H. Kinder, S. Messoloras, R.C. Newman, R.J. Stewart, J.G. Wilkes// Phil. Mag. B 59(5), p. 499 (1989).

28. K. Suneoka, N. Ikeda, T. Yamamoto, S. Kobayashi // J. Appl. Phys. 74(9), p. 5437 (1993). 